\title{
A Practical Approach for Simultaneous Estimation of Light Source Position, Scene Structure, and Blind Restoration Using Photometric Observations
}

\author{
Swati Sharma ${ }^{1,2}$ and Manjunath V. Joshi ${ }^{3}$ \\ ${ }^{1}$ Laboratoire d'Imagerie et de Neurosciences Cognitives, UMR CNRS-ULP 7191, 67000 Strasbourg, France \\ ${ }^{2}$ Laboratoire des Sciences de l'Image, de l'Informatique et de la Télédétection, UMR CNRS-ULP 7005, 67412 Illkirch Cedex, France \\ ${ }^{3}$ Dhirubhai Ambani Institute of Information and Communication Technology, Gandhinagar 382007, Gujarat, India
}

Correspondence should be addressed to Swati Sharma, swati.sharma@linc.u-strasbg.fr

Received 26 September 2007; Revised 15 February 2008; Accepted 2 April 2008

Recommended by Hubert Cardot

Given blurred observations of a stationary scene captured using a static camera but with different and unknown light source positions, we estimate the light source positions and scene structure (surface gradients) and perform blind image restoration. The images are restored using the estimated light source positions, surface gradients, and albedo. The surface of the object is assumed to be Lambertian. We first propose a simple approach to obtain a rough estimate of the light source position from a single image using the shading information which does not use any calibration or initialization. We model the prior information for the scene structure as a separate Markov random field (MRF) with discontinuity preservation, and the blur function is modeled as Gaussian. A proper regularization approach is then used to estimate the light source position, scene structure, and blur parameter. The optimization is carried out using the graph cuts approach. The advantage of the proposed approach is that its time complexity is much less as compared to other approaches that use global optimization techniques such as simulated annealing. Reducing the time complexity is crucial in many of the practical vision problems. Results of experimentation on both synthetic and real images are presented.

Copyright (c) 2008 S. Sharma and ManjunathV. Joshi. This is an open access article distributed under the Creative Commons Attribution License, which permits unrestricted use, distribution, and reproduction in any medium, provided the original work is properly cited.

\section{INTRODUCTION}

Photometric stereo has been used by many researchers for recovering the shape of the object and the albedo. Here, the shading cue is used for inferring the shape of the object. Authors in [1] propose two algorithms for robust shape estimation for photometric stereo. They combine finite triangular surface model and the linearized reflectance image formation model to express the image irradiance. Chen et al. [2] recover the albedo values for color images using photometric stereo. In [3-5], the authors use a calibrating object of known shape and constant albedo to establish a nonlinear mapping between the image irradiance and shape of the object in the form of a lookup table. For photometric stereo, a neural network-based approach is presented in [6] for a rotationally symmetric object with nonuniform reflectance. Authors in [7] obtain shape from photometric stereo images with unknown light source positions. However, they do not attempt to recover the light source positions. Basri et al. [8] attempt to recover the surface normal in a scene using the images produced under general lighting condition. They assume the light sources to be isotropic and distantly located from the object, assume a combination of point sources, extended sources, diffused lighting, and represent the general lighting conditions using low-order spherical harmonics. In [9], a method to obtain absolute depth from multiple images based on solving a set of linear equations is proposed. This method is applicable to a wide range of reflectance models. Another approach for photometric stereo that is based on the optical flow is presented in [10]. The input images are matched through an optical flow and the resulting disparity field is then used to obtain structure from motion which does not require the reflectance map information. Photometric stereo has also been applied to the analysis 
and description of surface structures in [11-14]. It has also been applied to the problems of machine inspections [15] and identification of machined surfaces [16]. In [17], graph cuts minimization technique has been used for estimation of the surface normals using photometric stereo. They use the ratio of two images in order to cancel out the albedo in the image irradiance equation and get the initial estimates of the surface normal which are required to define the energy functions. Graph cuts are then used for optimization. Although, authors in $[7,8]$ obtain the shape of the object without the knowledge of the light source position they do not consider the blur in the observations. In all these methods, the researchers do not consider the effect of blur while solving the problem of photometric stereo. In practice, the observations are often blurred due to camera jitter or outof-focus blur. Joshi and Chaudhuri [18] address the problem of simultaneous estimation of the scene structure and restore the images considering blurred photometric observations. They recover the surface gradients and the albedo and also perform blind image restoration. The surface gradients and the albedo are modeled as separate Markov random fields (MRFs), and a suitable regularization scheme is used to estimate the different fields as well as the blur parameter. However, they use simulated annealing for optimization which is very time-consuming and takes hours to reach the global minima. Also, the light source positions are assumed to be known. Sharma and Joshi [19] use graph cuts for superresolving the image and scene depth using photometric cue. However, they do not consider blur on the observations and use known light source directions. In this paper, we do not address the superresolution problem, but we estimate the scene structure, light source position, and perform blind image restoration.

Most of the researchers, while using shape from shading and photometric stereo, assume that the light source positions are known. However, in a practical scenario, the images are captured without any knowledge of the position of the light source (with respect to some reference plane). We now discuss briefly some of the research works that have been carried out on the estimation of position of the light source. The problem of obtaining the light source position from a single image was first addressed in [20] where the solution is obtained using the derivative of the image intensity along several directions. The authors in [21] present two schemes for estimating the illuminant direction from a single image. One method is based on local estimates for smooth patches. The second method uses shading information from image contours. In [22], a scheme which is based on the concept of critical points in the image for extracting multiple illuminant directions from the image of a sphere of known size is proposed. Two methods for estimating the surface reflectance property of an object as well as the position of a light source from a single view without the distant illumination assumption are proposed in [23]. Given an image and a 3D geometric model of an object with specular reflection as inputs, the first method estimates the light source position by fitting to the Lambertian diffuse component, while separating the specular and diffuse components by using an iterative relaxation scheme. The second method extends the first method by using specular component image as input, which is acquired by analyzing multiple polarization images taken from a single view. The authors in [24] combine information both from the shading of the object and from the shadows cast on the scene to estimate the position of multiple illuminants of a scene. In [25], a scheme for locating multiple light sources and estimating their intensities from a pair of stereo images of a sphere is discussed. The surface of the sphere is assumed to have both Lambertian and specular properties. In [26], a method is presented for calibrating multiple light source locations in $3 \mathrm{D}$ using captured images. This method uses three spheres at known relative positions which are used for calibrating the light source directions. In [27], a fully automatic algorithm for estimating the projected light source direction from a single image is presented. The algorithm consists of three stages. First, the potential occluding contours using color and edge information are selected, and then for each contour the light source direction is estimated using a shading model. In the final stage, the results from the estimations are fused together in a Bayesian network to arrive at the most likely light source direction. The approaches proposed in $[25,26]$ use calibration to find the light source position, which is a difficult task.

In this paper, we first propose a simple approach for obtaining the rough estimates of light source position using a single image. We assume a point light source and one light source direction for each captured image. We thus estimate the light source position for each observation in the photometric stereo setup. It may be mentioned that the proposed approach for light source direction does not use any calibration as used by many of the other researchers. We then estimate the scene structure and the blur parameter and restore the image. The blur function is modeled as Gaussian and the surface gradients are modeled as separate Markov random fields (MRFs) with edge preservation and suitable regularization is used. A cost function that consists of a data fitting term and other constraint terms is formulated and graph cuts approach is used for optimization to get the final solution. The light source position is also optimized for each of the captured image. We would like to mention here that we do not optimize for albedo assuming that it, as a smooth field and a simple sharpening filter, is used to remove the effect of blurring from the albedo field. Although the problem of blind restoration and shape estimation from blurred photometric observations is solved in [18], they use known light source positions and do not estimate them in their formulation. Also, they use simulated annealing for optimization which is computationally very taxing. In our formulation, we use graph cuts with proper choice of label set to considerably reduce the convergence time. It may be mentioned here that although simulated annealing yields global minima irrespective of the nature of cost function, the solution obtained using graph cuts is near the optimal solution [28] with computational complexity much less than simulated annealing. In a practical scenario, time complexity is crucial. For instance, if we consider an assembly line where an object has to be moved from one place to another (industrial inspection), the requirement 


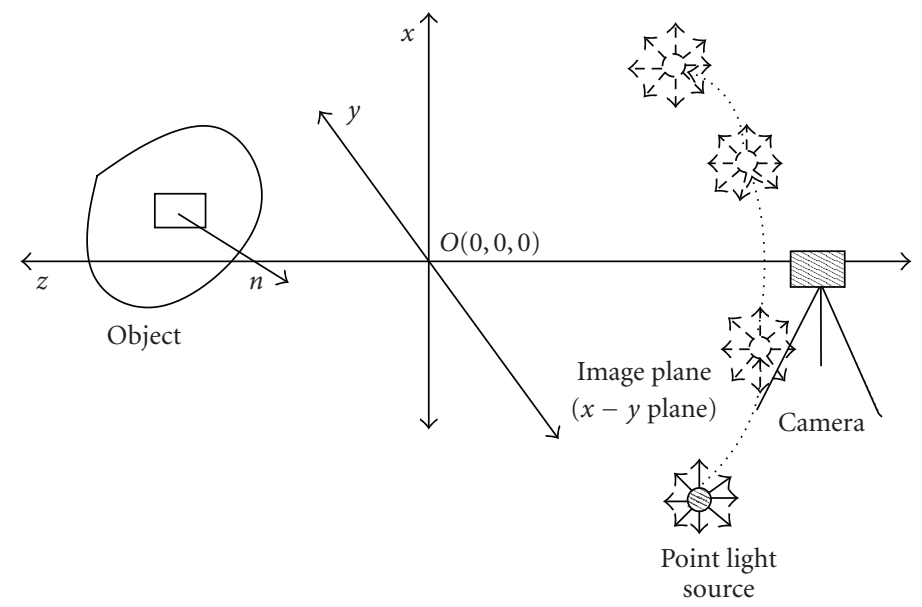

FIGURE 1: Observation system for photometric stereo.

is to calculate the depth fast enough so that the assembly line functions smoothly, with a slight compromise on the high accuracy. In such situations, near global optimization methods, such as graph cuts, are useful. It is interesting to note that the rough estimates of the proposed light source position approach serve as better initial estimates for graph cuts to reach near optimum result quickly.

It may also be mentioned here that uncalibrated photometric stereo may be used to find the surface gradients and albedo along with the light source directions and intensities. However, there is an ambiguity in the estimated values since these quantities can be determined only up to an arbitrary invertible matrix $[29,30]$. The proposed approach does not suffer from such a problem. Also, it uses a simple shading effect which forms the critical boundary in order to obtain the initial estimate.

The rest of the paper is organized as follows. In Section 2, we discuss the basic photometric stereo approach for shape (depth) estimation. Next, we explain the forward model for formation of blurred images in Section 3. Section 4 describes the proposed approach for light source direction estimation. A brief overview of the graph cuts optimization method is presented in Section 5. Section 6 deals with the proposed approach for simultaneous estimation of scene structure, light source direction, and blind image restoration. We present the results of experimentation for light source direction estimation, depth estimation, and blind restoration of images in Section 7. The paper is concluded with a short discussion in Section 8.

\section{PHOTOMETRIC STEREO}

Photometric stereo is a method for estimating the 3D shape of an object. It requires several images of a stationary object that are captured using a stationary camera with different light source positions. Figure 1 shows the observation system for photometric stereo, in which the object is placed at a fixed distance from the camera, and the light source is moved to different positions. For each position of the light source an image is captured, thus obtaining a set of images as observations. If a Lambertian surface is assumed, the image irradiance equation relating the surface gradients and image intensity can be written as

$$
\begin{aligned}
& E(x, y)=\rho(x, y) \hat{n}(x, y) \cdot \hat{s}, \\
& E(x, y)=\frac{\rho(x, y)\left(p(x, y) p_{s}+q(x, y) q_{s}+1\right)}{\sqrt{p(x, y)^{2}+q(x, y)^{2}+1} \sqrt{p_{s}^{2}+q_{s}^{2}+1}},
\end{aligned}
$$

where $p(x, y), q(x, y)$ are the surface gradients in $x$ and $y$ directions, respectively. Here $\rho(x, y)$ represents the albedo, which is nothing but the fraction of light reflected from the surface at a point $(x, y)$ and its value lies between 0 and 1. $\hat{n}(x, y)$ denotes the surface normal given by $(-p(x, y),-q(x, y), 1) /\left(\sqrt{p(x, y)^{2}+q(x, y)^{2}+1}\right)$ and $E(x, y)$ is the image irradiance (or image intensity) at point $(x, y)$ in the image. $\hat{s}=\left(-p_{s},-q_{s}, 1\right) /\left(\sqrt{p_{s}^{2}+q_{s}^{2}+1}\right)$ is a unit vector in the direction of the light source.

The surface gradients and albedo at a point are related to the intensity at that point according to (1). Since there are three unknowns $p(x, y), q(x, y), \rho(x, y)$, it is possible to obtain a unique solution using linearly independent equations. In real scenario, due to erroneous observations, the equations may be inconsistent, and hence one needs to capture more than three images with different light source positions and obtain the surface gradients and albedo by solving the overdetermined set of equations using the least squares (LS) method. Once the surface gradients are known, an iterative method can be used to obtain the depth map [31].

\section{FORWARD MODEL}

Equation (1) relates the true surface gradients and albedo when we assume that the observations are not blurred. However, due to the faulty focus settings of the camera, the observations are often blurred. If the effect of blur and noise 


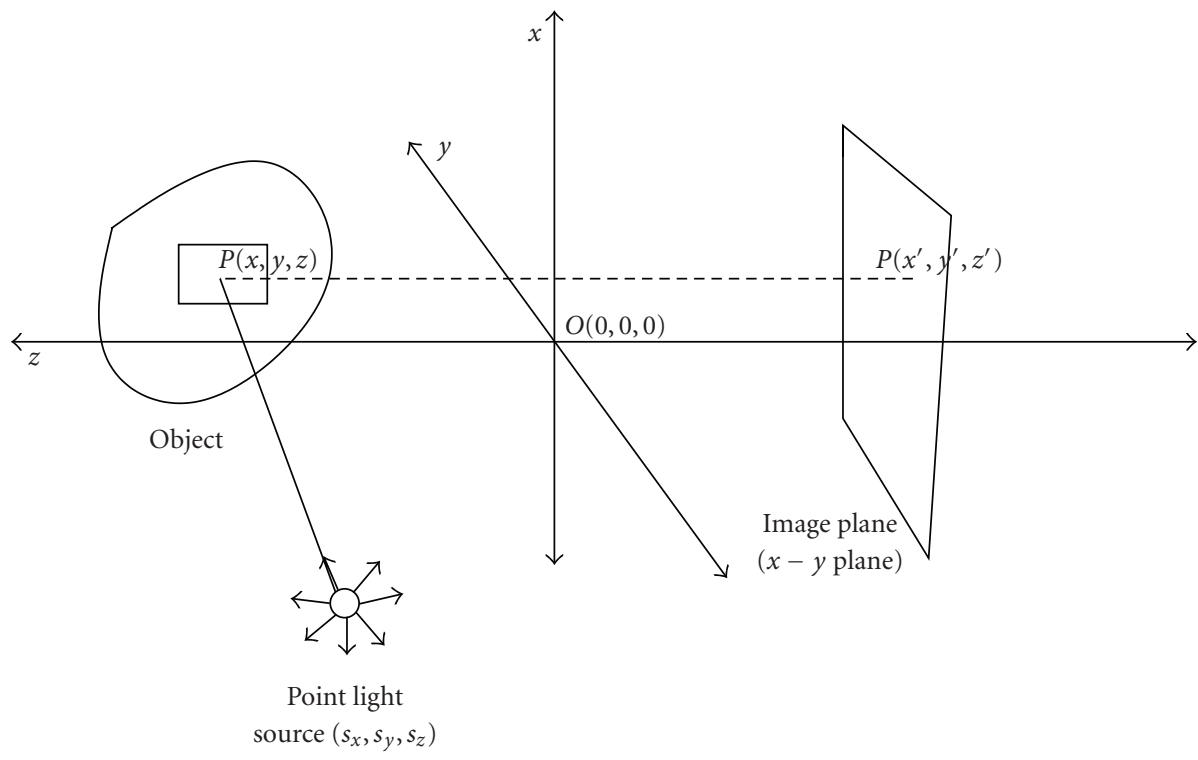

FIGURE 2: Experimental setup for estimating illuminant position. $P(x, y, z)$ is a point on the object that is projected onto the image plane at point $P\left(x^{\prime}, y^{\prime}, z^{\prime}\right)$.

is considered, then the image formed for the $m$ th light source position can be written as [18]

$$
g_{m}(x, y)=h(x, y) * E_{m}(x, y)+w_{m}(x, y), \quad m=1, \ldots, K
$$

where $h(x, y)$ represents the two-dimensional point spread function (PSF) of the camera, and $w_{m}(x, y)$ is the independent and identically distributed (i.i.d) additive noise, and $K$ denotes the number of blurred observations considered. Since, there is no relative motion between the camera and the object, the PSF remains same for all the observations. We also assume that the blur is space-invariant, and hence a single blur mask is assumed for the entire observed image. We also assume that there is no chromatic aberration due to the camera lens.

Now, let $E_{m}$ be a vector containing the unblurred intensity values of the $m$ th image of size $M \times N$ arranged in lexicographical order. $E_{m}$ is a function of $\rho, p, q$, and $\hat{s}_{m}$ which are the true values of the surface gradients, albedo, and the light source position. If $g_{m}$ represents the corresponding observation vector, (2) can be written as

$$
g_{m}=H(\sigma) E_{m}\left(\rho, p, q, \hat{s}_{m}\right)+w_{m}, \quad m=1, \ldots, K,
$$

where $H(\sigma)$ is the $M N \times M N$ matrix and $\sigma$ is the blur parameter. The blur is assumed to be due to the fact that the camera is out of focus. This can be modeled by a pillbox blur or by a Gaussian PSF characterized by the parameter $\sigma$ [32]. In our work, we assume Gaussian PSF with blur parameter $\sigma$. Now, the problem is to estimate the light source positions, the surface gradients, the albedo, and blur parameter given the observations. This is definitely an ill-posed problem and it requires the use of regularization to obtain better estimates.

While solving for the surface gradients and albedo using (1), one needs to know the light source direction. In a practical scenario, these are not known. In the following section, we discuss a simple approach for obtaining rough estimates of light source positions.

\section{PROPOSED APPROACH FOR INITIAL ESTIMATES OF LIGHT SOURCE POSITIONS}

Here, we discuss a simple shading-based method that uses the position of the critical boundary formed on the image of the object being imaged to estimate the light source position. The critical boundary is defined as that boundary beyond which the imaged object is not visible in the image due to the position of the light source. We assume that there is no selfocclusion and such a boundary exists due to the light source position. A single light source position is estimated for each of the blurred observations. We assume a point light source and an orthographic projection is assumed eliminating the need for geometric correction.

In this section, we use a different convention to represent the light source positions. The light source position is estimated with respect to a coordinate system. Let the vector $\left(s_{x}, s_{y}, s_{z}\right)$ represent the true light source position in the coordinate system. In the notation used in Section 2 , the unit light source vector is represented as $\left(-p_{s},-q_{s}, 1\right)$. Thus, we have the relation

$$
\begin{aligned}
& \frac{p_{s}}{\sqrt{p_{s}^{2}+q_{s}^{2}+1}}=\frac{-s_{x}}{\sqrt{s_{x}^{2}+s_{y}^{2}+s_{z}^{2}}}, \\
& \frac{q_{s}}{\sqrt{p_{s}^{2}+q_{s}^{2}+1}}=\frac{-s_{y}}{\sqrt{s_{x}^{2}+s_{y}^{2}+s_{z}^{2}}}, \\
& \frac{1}{\sqrt{p_{s}^{2}+q_{s}^{2}+1}}=\frac{s_{z}}{\sqrt{s_{x}^{2}+s_{y}^{2}+s_{z}^{2}}} .
\end{aligned}
$$


Figure 2 shows the position of the camera, the object, and the light source with respect to the coordinate system. Both the camera and the light source are placed in front of the object. We use simple geometry to find the light source position. The shading-based method for estimating the light source position is based on the fact that the critical boundary moves whenever the position of the light source changes. At the critical boundary on the image plane, a ray of light emanating from the light source becomes tangential (as the object is not visible in the image beyond that boundary). We refer to the coordinates of the image points on the end points of the critical boundary as critical points. If the critical points are known, then the tangents drawn at those points intersect at the point where the point light source is located. We use a simple binary thresholding followed by edge detection to obtain the critical boundary. Figure 3 illustrates the geometry used for the proposed method. The figure shows the tangents on the critical boundary and the light source position, given by the intersection of the tangents to the circle at the critical points. The dark portion of the figure shows the portion of the object beyond the critical boundary, which is not visible in the image. The light sources thus estimated for each observation are refined using the graph cuts optimization. It may be noted that we obtain the light source position using geometry on the image which lies on the $x-y$ plane, only the $x$ and $y$ coordinates of the light source direction can be estimated using our approach. The obtained coordinates are normalized to get the direction vector. We represent these as $s_{x}^{\prime}$ and $s_{y}^{\prime}$. The shading-based method can be summarized as follows.

(1) The given image is thresholded into two regions, depending on whether the portion of the object being imaged is visible in the image or not. We use the "watershed" function available in MATLAB to segment the object from the background.

(2) Edges are extracted from the image to get the critical boundary.

(3) Next, a best fit circle in the least square sense is estimated using the points on the critical boundary.

(4) Two tangents are drawn, one on each of the critical points of the critical boundary.

(5) The point of intersection of these tangents gives $x$ and $y$ coordinates of the light source position.

The rough estimates of the light source positions obtained from the blurred observations are used to obtain the initial values of $p, q$, and $\rho$ (using the least squares method as mentioned in Section 2), thus ensuring better initial estimates that aid in the quick convergence of the optimization using graph cuts. However, while using (1) to find the surface gradients and albedo, the $z$ coordinate of the light source position is initialized as follows. A small value $\varepsilon$ is subtracted from $s_{x}^{\prime}$ and $s_{y}^{\prime}$ such that the relation $\left(s_{x}^{\prime}-\varepsilon\right)^{2}+\left(s_{y}^{\prime}-\varepsilon\right)^{2}+$ $s_{z}^{\prime 2}=1$ is satisfied. We subtract a small value $\varepsilon$ from the values $s_{x}^{\prime}$ and $s_{y}^{\prime}$ (estimated geometrically from the image) as these values are already close to the true values. Since $s_{x}^{\prime}$ and $s_{y}^{\prime}$ are already normalized and close to the normalized true values

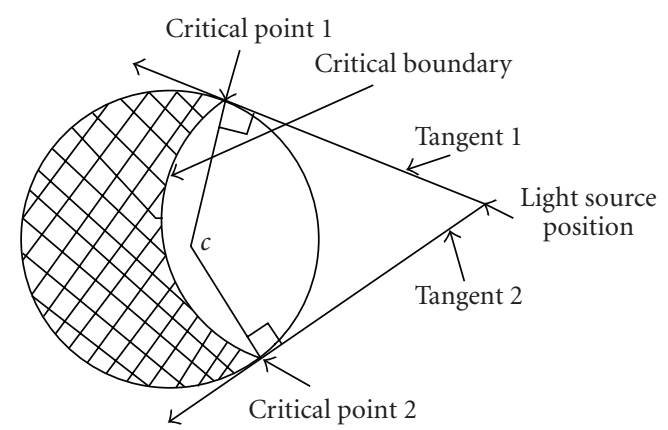

FIgURE 3: Illustration of the geometry used by the method. Also, shown are tangents on the critical boundary and the light source position (as the intersection of the two tangents at the critical points).

$s_{x} /\left(\sqrt{s_{x}^{2}+s_{y}^{2}+s_{z}^{2}}\right)$ and $s_{y} /\left(\sqrt{s_{x}^{2}+s_{y}^{2}+s_{z}^{2}}\right)$, this step is required so that the estimated initial light source position becomes a valid direction.

\section{INTRODUCTION TO GRAPH CUTS}

Many researchers use global optimization techniques such as simulated annealing for minimization of energy functions. Although, simulated annealing is theoretically capable of finding the global minima of an arbitrary energy function, it is computationally very expensive and hence practically not feasible. Recently, algorithms have been proposed for optimization using graph cuts which guarantee that the solution obtained either reaches the global optimum or reaches local minima close to the global minimum [28] quite fast.

One of the most widely used energy function in the graph cuts framework is as follows [28]:

$$
\begin{aligned}
E(f)= & \sum_{(x, y) \in S} \operatorname{Data}(f(x, y)) \\
& +\sum_{(x, y),(u, v) \in N} V_{(x, y),(u, v)}(f(x, y), f(u, v)) .
\end{aligned}
$$

$\operatorname{Data}(f(x, y))$ is a function derived from the observed data that measures the cost of assigning the label $f(x, y)$ to the pixel $(x, y) \in \delta, \&$ being the image grid. The label may represent an image intensity for a restoration problem or may be a surface gradient while estimating shape. $V_{(x, y),(u, v)}(f(x, y), f(u, v))$ is the term used to incorporate the spatial smoothness. This measures the cost of assigning the labels $f(x, y)$ and $f(u, v)$ to two adjacent pixels at $(x, y)$ and $(u, v)$. This is also the typical energy function that uses MRF modeling. Graph cuts can be used for minimization of only a certain type of energy functions. Minimization via graph cuts is possible only if the cost function is graph representable. It has been proved that an energy function is graph representable provided the energy function satisfies the regularity condition [33].

Minimization of an energy function by graph cuts is basically finding that cut on the graph which has the minimum cost. Such algorithms are called min-cut/max-flow 
algorithms. Global minimization of these energy functions is NP-hard even in the simplest discontinuity-preserving case. In [28], two min-cut/max-flow algorithms, $\alpha-\beta$ swap and $\alpha$ expansion have been proposed. It has been proved that iteratively running the expansion algorithm produces approximate solutions within a factor of two of the global minima for a multilabel case provided that the smoothness term $V_{(x, y),(u, v)}(f(x, y), f(u, v))$ is a metric. This motivates us to use graph cuts as an optimization method in our work.

\section{ESTIMATION OF SCENE STRUCTURE, LIGHT SOURCE POSITION, AND BLIND RESTORATION}

In the following section, we explain how we solve our problem of estimating the light source directions, surface gradients, and the blur parameter.

\subsection{Data fitting term}

Since, we have many observations of the same stationary object captured with a stationary camera, the data fitting term (from (3)) can be written as

$$
\text { Dataterm }=\sum_{m=0}^{K-1}\left\|g_{m}-H(\sigma) E_{m}\left(\rho, p, q, s_{m}\right)\right\|^{2},
$$

where the symbols have their usual meaning. In this case, the variables are surface gradients, that is, $p(x, y)$ and $q(x, y)$, albedo $\rho(x, y)$ at every pixel $(x, y)$ of the image. Also, the illuminant position $\widehat{s}_{m}$ is unknown but is the same for the entire image. In order to simplify calculations, we parameterize the point light source in terms of the tilt $\left(\tau_{m}\right)$ and slant $\left(\gamma_{m}\right)$ angles. Then, the unit vector in the illuminant direction is

$$
\begin{aligned}
\hat{\boldsymbol{s}}_{m} & =\left(\hat{\boldsymbol{s}}_{x_{m}}, \hat{\boldsymbol{s}}_{y_{m}}, \hat{\boldsymbol{s}}_{z_{m}}\right) \\
& =\left(\cos \left(\tau_{m}\right) \sin \left(\gamma_{m}\right), \sin \left(\tau_{m}\right) \sin \left(\gamma_{m}\right), \cos \left(\gamma_{m}\right)\right) .
\end{aligned}
$$

This a multilabel minimization problem with a number of unknowns.

The energy function should satisfy the regularity condition so that it can be minimized using graph cuts formulation. Applications of graph cuts generally use the data term that is a function of a single pixel [34] since a function of a single variable is always regular [33].

Consider the data fitting term for a particular pixel $(x, y)$ of the images. Equation (6) can be written as

$$
\operatorname{Dataterm}(x, y)=\sum_{m=0}^{K-1}\left[g_{m}(x, y)-\sum_{i=-u}^{u} \sum_{j=-v}^{v} h(i, j) F_{m}(x, y)\right]^{2},
$$

where

$$
F_{m}(x, y)=E_{m}\left(\rho(x-i, y-j), p(x-i, y-j), q(x-i, y-j), s_{m}\right),
$$

where $h$ is an $S \times T$ blurring mask, $u=(S-1) / 2$, and $v=(T-1) / 2$. Since the blurring function $H(\sigma)$ operates on more than one pixel, the data term is not regular. In order to use the graph cuts formulation, we apply valid mathematical approximations to the data fitting term such that the data term becomes a function of a single pixel. For each pixel $(x, y)$, we consider the terms not depending on $(x, y)$ as constant for a particular optimization step. Then (8) can be rewritten as

$$
\begin{gathered}
\operatorname{Dataterm}(x, y)=\sum_{m=0}^{K-1}\left[g_{m}(x, y)-\left(h(0,0) F_{m}(x, y)+C\right)\right]^{2}, \\
C=\sum_{i=-u}^{u} \sum_{j=-v}^{v} h(i, j) F_{m}(x, y), \quad i \neq 0, j \neq 0
\end{gathered}
$$

\subsection{Prior modeling}

We model the prior information of the surface gradients as separate Markov random fields (MRFs). By using the MRF prior, the spatial dependency between the neighboring pixels can be easily accounted. Generally, the depth variation of an object is smooth with occasional discontinuities representing sudden change in depth. We capture this relationship by using the smoothness term with discontinuity preservation for edges. In this case, a truncated linear prior as defined in [28] is used. The discontinuity preservation depends on the choice of parameter $T$. This prior is piecewise smooth, and hence it ensures that the solution does not become over smooth, and discontinuities are preserved. The smoothness term for two neighboring pixels $(x, y)$ and $(k, l)$ is given by the following expression:

$$
V_{(x, y),(k, l)}(f(x, y), f(k, l))=\min (|f(x, y)-f(k, l)|, T),
$$

where $T$ is a positive constant. The smoothness term satisfies the regularity condition if it is a metric. It can be easily verified that (12) satisfies the conditions of a metric. Here, $f(x, y)$ is the label assigned to the pixel $(x, y)$. So, $f(x, y)$ can be either $p(x, y)$ or $q(x, y)$. We use the following truncated linear prior for $p$ and $q$ :

$$
\begin{aligned}
U(t)=\lambda_{t} \sum_{x=1}^{M} \sum_{y=1}^{N}[ & \min \left(t(x, y)-t(x-1, y), T_{t}\right) \\
& \left.+\min \left(t(x, y)-t(x, y-1), T_{t}\right)\right],
\end{aligned}
$$

where $t=p$ or $q$

\subsection{Source position direction constraint}

Since we estimate the normalized light source direction, the estimated value of the illuminant position should satisfy

$$
\|s\|^{2}=1,
$$

where $s=\left(s_{x}, s_{y}, s_{z}\right)$. This ensures that the light source position is a unit vector in the direction of the source. This constraint is used while optimizing to ensure better convergence of the light source positions. 


\subsection{Total cost function}

Since we use a regularization-based approach, the total cost function can be obtained by combining the data term, smoothness term, and the source position constraint. Thus using (10), (13), and (14), we can express the total cost function as

$$
\begin{aligned}
\varepsilon=\sum_{m=0}^{K-1} \sum_{\text {over all } x ; y}[ & g_{m}(x, y) \\
& -\left(h(0,0) E_{m}\left(\rho(x, y), p(x, y), q(x, y), s_{m}\right)\right. \\
& +C)]^{2}+U(p)+U(q)+\left(s^{2}-1\right)^{2}
\end{aligned}
$$

In our implementation, we optimize one variable at a time keeping the others constant. For example, the cost is minimized first using $p$ values, keeping the values of $q, \tau_{m}, \gamma_{m}$, and $\sigma$ constant. Using the optimized values of $p$, we minimize for $q$, keeping the other variables unchanged. This is repeated in each cycle for all the variables until convergence is reached. It may be mentioned here that $p, q$ are all matrices. $\gamma_{m}$ and $\tau_{m}$ are real values corresponding to a particular source position and $\sigma$ is also a real value. As already mentioned, we use the albedo values that are unblurred using a simple high pass filter to reconstruct the restored images for each light source direction. The depth is estimated using the estimated $p$ and $q$ values [31].

\subsection{Choice of the label set}

Graph cuts require a discrete label set. Many of the proposed methods use graph cuts because optimization use integer labels, for example, see [35]. In our case, we use discrete floating point labels. Knowing the initial light source position estimates, one can obtain the initial estimates for $p$, $q$, and albedo using an LS approach. Based on the frequency distribution (histogram) of $p$ and $q$ labels, it is possible to quantize the entire range of continuous labels in a nonuniform fashion to get a discrete label set. The nonuniform quantization is done so that maximum number of labels (discrete and integer) is assigned to that subrange which has a higher probability. For $\tau$ and $\gamma$, the set of labels is selected by trial and error around the initially obtained values. The number of labels, in this case, is directly related to the precision. As the chosen number of labels is increased, more accurate estimates may be obtained with a slight increase in computational complexity.

\section{EXPERIMENTAL RESULTS}

In this section, we present some of our experimental results for the proposed approach to recover the light source positions, depth estimation (using the estimated surface gradients), and blind restoration. Experimental results are shown for synthetically generated images as well as for real images.

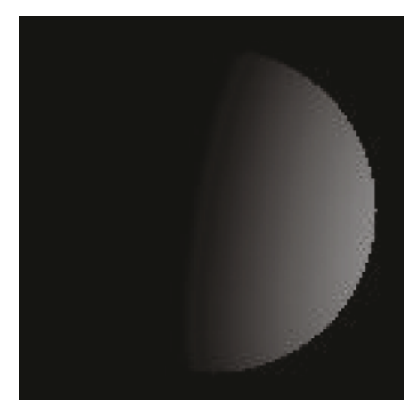

(a)

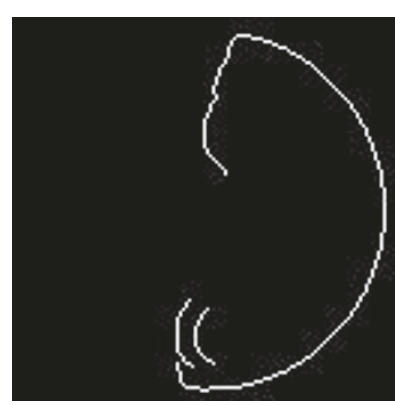

(b)
FIGURE 4: (a) Synthetically generated hemisphere image with light source position $(0.1545,0.9755,0.1564)$ and (b) the corresponding edge image.

\subsection{Experimental results on initial estimates of light source positions}

We first consider the experimentation for estimating the light source position using the proposed shading-based method. An image of a hemisphere with known light source position is synthesized. While conducting the experiment, we assume that the light source position is unknown. Figure 4(a) shows the image of the hemisphere with normalized $x$ and $y$ coordinates of the light source direction as $(0.1545,0.9755)$, and the corresponding edge image is shown in Figure 4(b). We use a simple canny edge detection technique to obtain the edge image. Since the image is a circle, the line joining the center of the image to the critical points will be perpendicular to the tangents at these points, and the intersection point of these tangents gives the $x$ and $y$ coordinates of the light source position. The estimated values of the $x$ and $y$ coordinates of light source position in this case are $(0.1592,0.9872)$ which are quite close to the true estimate. Table 1 shows the actual and estimated values of $x$ and $y$ coordinates of the light source direction for the images of the hemisphere generated using different light source directions. We next consider a real image with unknown light source directions where the critical boundary may not be a smooth curve. Figure 5(a) shows the image of a soft toy "Jodu" captured with some unknown light source position and the corresponding edge image is shown in Figure 5(b). In this case, in order to obtain the light source position, we fit a circle through the image points that lie on the critical boundary. Now, the two critical points are selected on this circle, and the point of intersection of the tangents at these points is the light source position. This experiment was repeated on a set of eight images of Jodu so that they can be used as the initial estimates for graph cuts optimization. In order to verify the correctness of the light source direction, we reconstruct the images using these estimated light source positions and the initial estimates of $p, q$, and $\rho$ obtained using them (refer to (1)). The reconstructed image displayed in Figure 5(c) has been shading very close to the displayed image in Figure 5(a). This indicates that these initial estimates of the light source position when further used in graph cuts optimization lead 


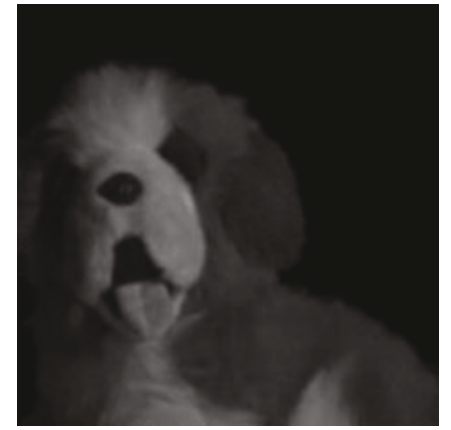

(a)

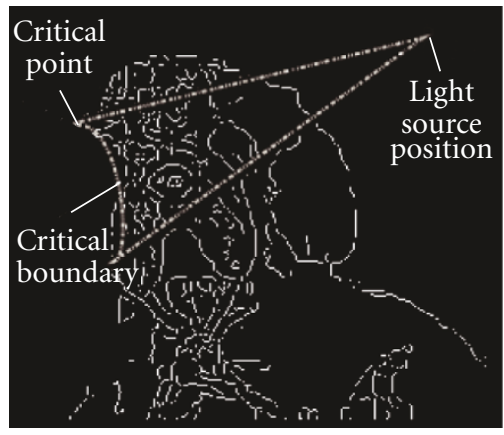

(b)

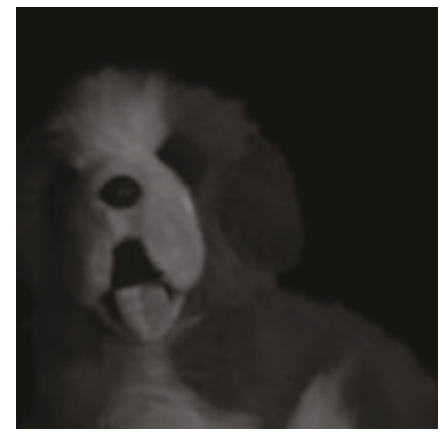

(c)

FIGURE 5: (a) Observed Jodu image with unknown light source position. (b) Edge image of Jodu with the same source position. Also shown in the figure is the circle fitted for the critical boundary and the light source position. (c) Reconstructed Jodu image with the initially estimated light source direction $(0.3821,0.7035,0.5992)$.

TABLE 1: Actual and estimated values of $x$ and $y$ coordinates of the light source position for the hemisphere image.

\begin{tabular}{lccc}
\hline \multicolumn{2}{l}{ Actual source position } & \multicolumn{2}{c}{ Estimated source position } \\
$x$ & $y$ & $x$ & $y$ \\
\hline 0.1545 & 0.9755 & 0.1592 & 0.9872 \\
0.2034 & 0.9568 & 0.2069 & 0.9874 \\
0.3716 & 0.8346 & 0.4172 & 0.9088 \\
0.2939 & 0.9045 & 0.2916 & 0.9566 \\
\hline
\end{tabular}

to convergence of the $x, y$, and $z$ coordinates of the light source positions.

\subsection{Experimental results on depth estimation and blind restoration of images}

In order to obtain the depth map and blind restoration of images, we need to estimate the surface gradients and the blur parameter given the blurred observations. Since the initial light source positions are already known, we obtain the initial $p, q$, and $\rho$ values which serve as initial estimates for optimization. As mentioned earlier, we do not optimize the albedo field. For the implementation, we use the graph cuts library provided by Kolmogorov [28, 33, 36]. Particularly, we use the expansion algorithm for the cost function minimization. As already discussed, we use a fixed set of labels for each of the entities $p, q$, light source position, and the blur parameter.

We first consider a synthetic image of a vase with a checkerboard pattern on it. Eight images each of size $128 \times$ 128 are generated with different light source positions using a computer program. In order to test our algorithm, we blur the vase images using a Gaussian blur kernel since the blur due to defocus can be modeled as Gaussian [32]. However, we assume that the blur is space invariant for our experiments. Since the defocus is assumed to be small, the blur parameter $(\sigma)$ of the Gaussian function is assumed to lie in the range $(0.5,1.5)$. For this experiment, the blur

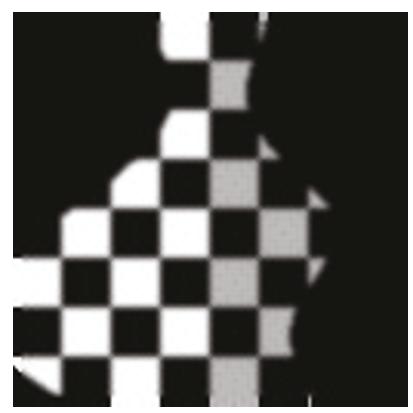

(a)

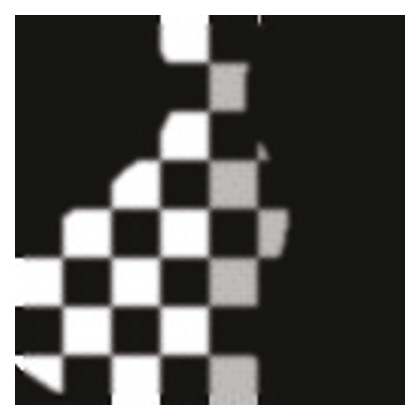

(b)
FIGURE 6: Synthesized vase images with source positions: (a) $(0.2995,0.4827,0.8230)$, (b) $(0.4379,0.4827,0.7585)$.

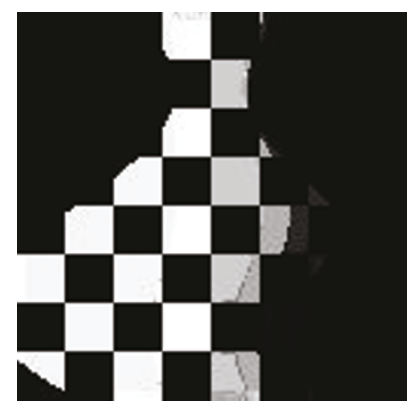

(a)

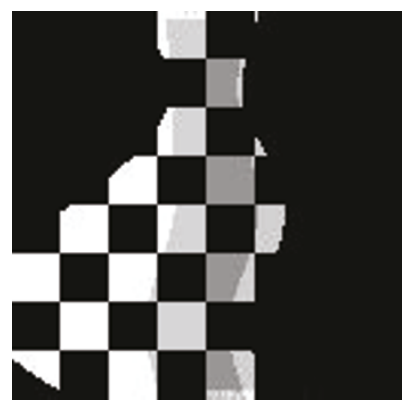

(b)
FIGURE 7: Restored vase images using the proposed approach for the observations in Figure 6. The estimated light source positions are $(0.3871,0.5492,0.7407)$ and $(0.4554,0.3778,0.8062)$, respectively.

parameter was chosen to be $\sigma=1$ and the kernel size was $7 \times$ 7. Figure 6 shows two of the observed vase images with true light source positions: Figure 6(a) $(0.2995,0.4827,0.8230)$ and Figure 6(b) $(0.4379,0.4827,0.7585)$. The blur parameter $\sigma$ estimated using our approach is 0.93 which is very close to the true value of $\sigma=1$. The number of labels for estimating the same was chosen as 10 . Figures $7(a)$ and 


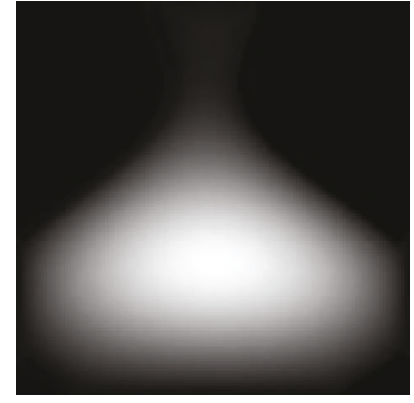

(a)

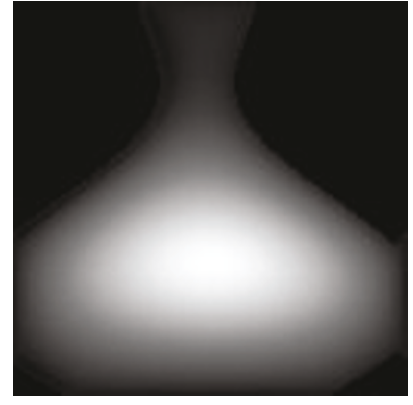

(b)

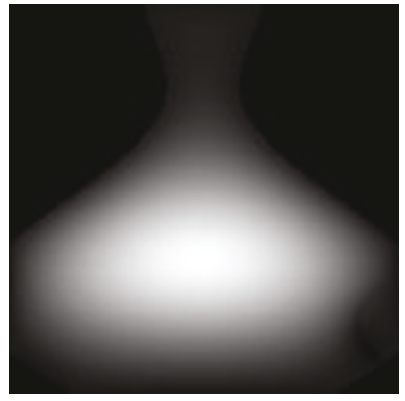

(c)

FIGURE 8: Depth map for vase (a) ground truth and obtained using (b) LS approach on blurred images and (c) proposed approach.

7(b) show the restored vase images after optimization with graph cuts. The two images have similar shading as given in Figures 6(a) and 6(b) indicating that the source positions estimated are close to the correct values. The sharp square patches with clear edge detail indicate that the images are well restored. Figures 8(a) and 8(b) show the ground truth for depth and that obtained using blurred images. The ground truth for the vase image is known since it is a synthetic image. Figure 8(c) displays the recovered depth map using the proposed approach. The depth map is shown as an intensity image that represents the depth values scaled in the range $0-255$. The scaling is done such that higher intensity pixels in the depth map represent points closer to the camera in the object.

For the vase image, we observed that the initial values of $p$ and $q$ lie in the range $(-4,0.6)$ and $(-0.2,0.3)$, respectively. Hence, depending on the frequency distributions of the respective entities, we used 388 and 350 labels for $p$ and $q$, respectively. The number of labels for both the tilt and slant angles of the light source position were chosen as 40 . The regularization parameters $\lambda_{p}$ and $\lambda_{q}$ for $p$ and $q$ fields (in (13)) were manually selected as 0.075 and 0.034 , respectively. The value of $T_{t}$ of the truncated linear prior was chosen to be 0.175 . These were chosen on a trial and error basis.

In order to test our algorithm on real images, we next consider the experimentation on two real image sets, Jodu and shoe. The light source positions are unknown for Jodu images but the same is available for shoe images. We slightly defocus the camera setting to obtain the blurred Jodu and shoe observations. In a real scenario, this is due to improper focus setting while using an inferior quality camera.

We first consider Jodu images. Two of the observed images, with unknown light source positions, are shown in Figures 9(a) and 9(b). Figures $10(a)$ and 10(b) show the restored Jodu images after optimization using our approach. In both cases, it can be clearly seen that the two images have been shading very similar to that displayed in Figures 9(a) and 9 (b), indicating that the estimated source positions are close to the true values. The reconstructed images are also sharper as compared to the blurred observations indicating that they are restored well. The blur parameter $\sigma$ estimated for this experiment was 0.84 .

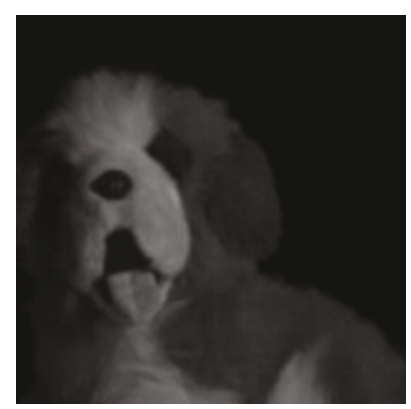

(a)

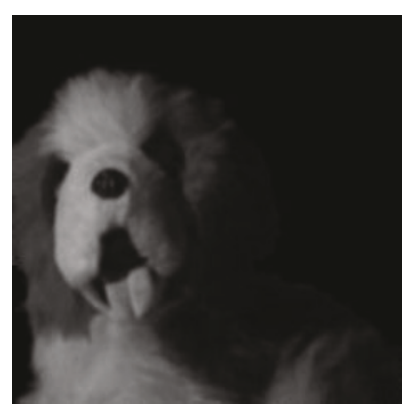

(b)
Figure 9: Observed Jodu images with unknown light source directions.

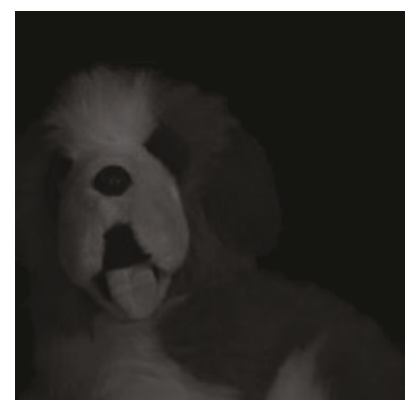

(a)

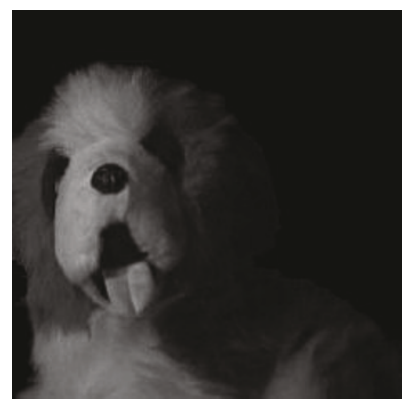

(b)
FIGURE 10: Reconstructed Jodu images after optimization using graph cuts. In this case, the estimated source positions are (a) $(0.4379,0.4827,0.7585)$, (b) $(-0.5428,-0.4823,0.6875)$.

The initialization for this experiment was kept as follows. Since the initial values of $p$ were in the range $(-1,1)$ and that for $q$ lies in the range $(-0.6,0.6)$, depending on the frequency distributions of the respective entities, we used 440 and 420 labels for $p$ and $q$, respectively. The other parameters $\lambda_{t}$ and $T_{t}$, where $t=p, q$, as well as the number of labels for tilt and slant angles of the light source position and the blur parameters were kept the same as the previous experiment, for both Jodu and shoe image sets. 


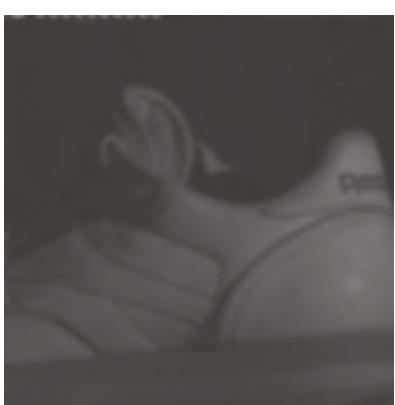

(a)

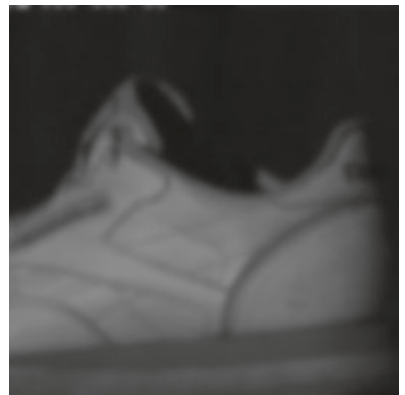

(b)
FIGURE 11: Observed shoe images with true light source directions (a) $(0.6736,0.3042,0.6736)$, (b) $(-0.6123,-0.3042,0.7297)$.

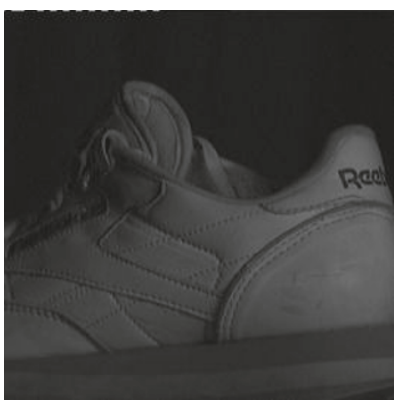

(a)

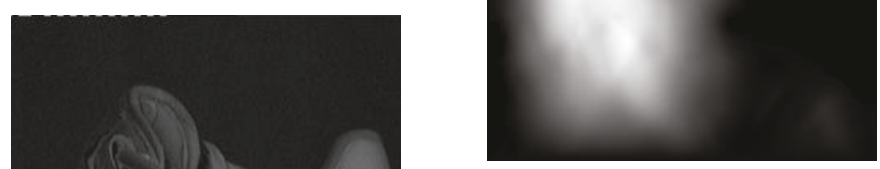

(a)

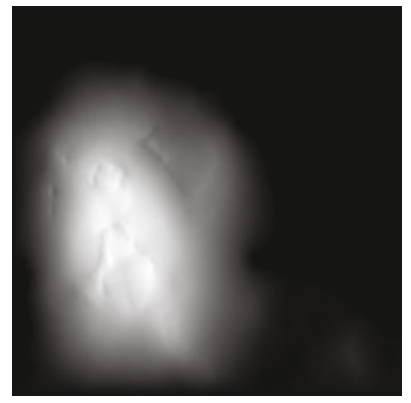

(b)
FIGURE 13: Depth map for Jodu obtained using (a) LS approach on blurred images, (b) proposed approach.

In order to compare the performance based on the quantitative measure, we use peak signal-to-noise ratio (PSNR) as a figure of merit for both the reconstructed images and the depth map. The expression for PSNR is given as follows:

$$
\mathrm{PSNR}=20 \log \left(\frac{255}{\sqrt{\mathrm{MSE}}}\right)
$$

where

$$
\text { MSE }=\frac{1}{M N} \sum_{x=0}^{M-1} \sum_{y=0}^{N-1}(I(x, y)-J(x, y))^{2}
$$

for two $M \times N$ images $I$ and $J$. Here $I$ is the true image and $J$ represents either the observed blurred image or the estimated one.

Table 2 shows the PSNR values for the blurred vase images and those obtained after using the proposed approach. The values are tabulated for vase intensity image with two different light source positions as well as for the depth. We can clearly see that with the graph cuts-based approach the PSNR improves for the restored images.

Since vase is a smooth image, the depth map reconstructed from the blurred images using the correct light source positions is close to the ground truth. Hence in case of the reconstructed depth map using the proposed approach, there is a slight decrease in the value of PSNR although perceptually it is close to the ground truth as is clearly seen in Figure 8(c). It may be mentioned here that we cannot compare PSNR for the restored Jodu and shoe images as well as their depth maps, since we do not have the ground truth.

We would also like to mention that our method works well for sphere-shaped objects (for, e.g., vase image) as the intensity variation in the depth map. This can be observed in Figure 14(b) indicating a better depth estimate.
TABLE 2: PSNR comparison for vase images. The (depth) row in the 政 


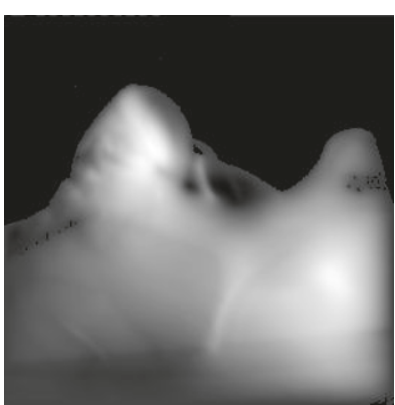

(a) (b)

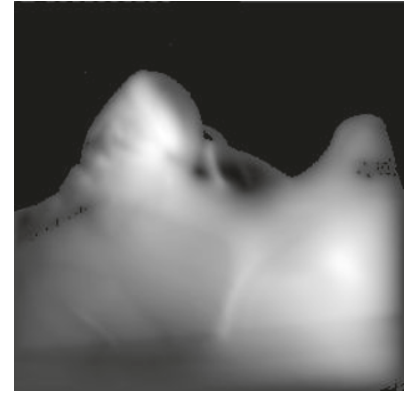

Figure 14: Depth map for shoe obtained using (a) LS approach on blurred images, (b) proposed approach.

method relies on fitting a circle on the critical boundary. However, in our experiments on arbitrary object shapes (Jodu and shoe), we found no convergence problems when we used the light source positions estimated using the proposed approach as initial estimates and then refined them using graph cuts.

We now compare the time complexity of our approach with that proposed in [18], where simulated annealing is used for optimization in order to preserve the discontinuities. The convergence time for the algorithm proposed in the paper was of the order of hours which makes the algorithm unfit in a practical scenario. Our approach, on the other hand, takes around 5-7 minutes for convergence. All experiments were performed on a $1.33 \mathrm{GHz}$ processor usin vase, Jodu, and shoe images of size $128 \times 128,234 \times 234$, and $265 \times 265$, respectively.

\section{CONCLUSIONS}

In this paper, we present a practical approach for photometric stereo. First, we propose a simple method to obtain rough estimates for light source position which does not require any calibration or initialization. We then use these initial estimates to obtain the light source positions, blur parameter, scene depth, as well as the restored images given only the blurred photometric observations. A proper regularization scheme is used for the same, and graph cuts were used for optimization. The advantage of the proposed approach is that we obtain the light source position, scene structure, and perform blind restoration given just the observations. The results show that the proposed approach produces results close to the desired solution. Results also show that the proposed approach is very fast as compared to other approaches that use global optimization techniques like simulated annealing. Thus our approach is useful in practical applications where computation time is a constraint.

\section{ACKNOWLEDGMENTS}

The authors would like to thank the reviewers for their constructive suggestions and comments. They also would like to thank Dr. André Jalobeanu, LSIIT, Université Louis
Pasteur (Illkirch, France), for his suggestions on improving the initial draft.

\section{REFERENCES}

[1] K. M. Lee and C.-C. J. Kuo, "Shape reconstruction from photometric stereo," in Proceedings of IEEE Computer Society Conference on Computer Vision and Pattern Recognition (CVPR '92), pp. 479-484, Champaign, Ill, USA, June 1992.

[2] C.-Y. Chen, R. Klette, and R. Kakarala, "Albedo recovery using photometric stereo approach," in Proceedings of the 16th International Conference on Pattern Recognition (ICPR '02), vol. 3, pp. 700-703, Quebec, Canada, August 2002.

[3] Y. Iwahori, R. J. Woodham, M. Ozaki, H. Tanaka, and N. Ishii, "Neural network based photometric stereo with a nearby rotational moving light source," IEICE Transactions on Information and Systems, vol. E80-D, no. 9, pp. 948-957, 1997.

[4] Y. Iwahori, R. J. Woodham, and A. Bagheri, "Principal component analysis and neural network implementation of photometric stereo," in Proceedings of the Workshop on PhysicsBased Modeling in Computer Vision, pp. 117-125, Cambridge, Mass, USA, June 1995.

[5] R. J. Woodham, "Gradient and curvature from the photometric-stereo method including local confidence estimation," Journal of the Optical Society of America A, vol. 11, no. 11, pp. 3050-3068, 1994.

[6] Y. Iwahori, R. J. Woodham, Y. Watanabe, and A. Iwata, "Selfcalibration and neural network implementation of photometric stereo," in Proceedings of the 16th International Conference on Pattern Recognition (ICPR '02), vol. 4, pp. 359-362, Quebec, Canada, August 2002.

[7] O. Drbohlav and R. Sara, "Unambiguous determination of shape from photometric stereo with unknown light sources," in Proceedings of the 8th IEEE International Conference on Computer Vision (ICCV '01), vol. 1, pp. 581-586, Vancouver, Canada, July 2001.

[8] R. Basri, D. Jacobs, and I. Kemelmacher, "Photometric stereo with general, unknown lighting," International Journal of Computer Vision, vol. 72, no. 3, pp. 239-257, 2007.

[9] J. J. Clark, "Active photometric stereo," in Proceedings of IEEE Computer Society Conference on Computer Vision and Pattern Recognition (CVPR '92), pp. 29-34, Champaign, Ill, USA, June 1992.

[10] J. R. A. Torreão, "A new approach to photometric stereo," Pattern Recognition Letters, vol. 20, no. 5, pp. 535-540, 1999.

[11] G. McGunnigle and M. J. Chantler, "Rotation invariant classification of rough surfaces," IEE Proceedings: Vision, Image and Signal Processing, vol. 146, no. 6, pp. 345-352, 1999.

[12] G. McGunnigle and M. J. Chantler, "Rough surface classification using point statistics from photometric stereo," Pattern Recognition Letters, vol. 21, no. 6-7, pp. 593-604, 2000.

[13] G. McGunnigle and M. J. Chantler, "Modelling deposition of surface texture," Electronics Letters, vol. 37, no. 12, pp. 749750, 2001.

[14] M. L. Smith, G. Smith, and T. Hill, "Gradient space analysis of surface defects using a photometric stereo derived bump map," Image and Vision Computing, vol. 17, no. 3-4, pp. 321332, 1999.

[15] P. Hansson and P. Johansson, "Topography and reflectance analysis of paper surfaces using a photometric stereo method," Optical Engineering, vol. 39, no. 9, pp. 2555-2561, 2000. 
[16] G. McGunnigle and M. J. Chantler, "Segmentation of machined surfaces," in Proceedings of the Irish Machine Vision and Image Processing Conference (IMVIP '01), pp. 200-207, Maynooth, Ireland, September 2001.

[17] T.-P. Wu and C.-K. Tang, "Dense photometric stereo using a mirror sphere and graph cut," in Proceedings of the IEEE Computer Society Conference on Computer Vision and Pattern Recognition (CVPR '05), vol. 1, pp. 140-147, San Diego, Calif, USA, June 2005.

[18] M. V. Joshi and S. Chaudhuri, "Joint blind restoration and surface recovery in photometric stereo," Journal of the Optical Society of America A, vol. 22, no. 6, pp. 1066-1076, 2005.

[19] S. Sharma and M. V. Joshi, "A practical approach for superresolution using photometric stereo and graph cuts," in Proceedings of the 18th British Machine Vision Conference (BMVC '07), Warwick, UK, September 2007.

[20] A. P. Pentland, "Finding the illuminant direction," Journal of the Optical Society of America, vol. 72, no. 4, pp. 448-455, 1982.

[21] Q. Zheng and R. Chellappa, "Estimation of illuminant direction, albedo, and shape from shading," IEEE Transactions on Pattern Analysis and Machine Intelligence, vol. 13, no. 7, pp. 680-702, 1991.

[22] Y. Zhang and Y.-H. Yang, "Illuminant direction determination for multiple light sources," in Proceedings of the IEEE Computer Society Conference on Computer Vision and Pattern Recognition (CVPR '00), vol. 1, pp. 269-276, Hilton Head Island, SC, USA, June 2000.

[23] K. Hara, K. Nishino, and K. Ikeuchi, "Light source position and reflectance estimation from a single view without the distant illumination assumption," IEEE Transactions on Pattern Analysis and Machine Intelligence, vol. 27, no. 4, pp. 493-505, 2005.

[24] Y. Wang and D. Samaras, "Estimation of multiple directional light sources for synthesis of mixed reality images," in Proceedings of the 10th Pacific Conference on Computer Graphics and Applications (PCCGA '02), pp. 38-47, Beijing, China, October 2002.

[25] W. Zhou and C. Kambhamettu, "Estimation of the size and location of multiple area light sources," in Proceedings of the 17th International Conference on Pattern Recognition (ICPR '04), vol. 3, pp. 214-217, Cambridge, Mass, USA, August 2004.

[26] M. W. Powell, S. Sarkar, and D. Goldgof, "A simple strategy for calibrating the geometry of light sources," IEEE Transactions on Pattern Analysis and Machine Intelligence, vol. 23, no. 9, pp. 1022-1027, 2001.

[27] P. Nillius and J.-O. Eklundh, "Automatic estimation of the projected light source direction," in Proceedings of the IEEE Computer Society Conference on Computer Vision and Pattern Recognition (CVPR '01), vol. 1, pp. 1076-1083, Kauai, Hawaii, USA, December 2001.

[28] Y. Boykov, O. Veksler, and R. Zabih, "Fast approximate energy minimization via graph cuts," IEEE Transactions on Pattern Analysis and Machine Intelligence, vol. 23, no. 11, pp. 12221239, 2001.

[29] H. Kong, P. Xu, and E.-K. Teoh, "Binocular uncalibrated photometric stereo," in Proceedings of the 2nd International Symposium on Advances in Visual Computing (ISVC '06), vol. 4291 of Lecture Notes in Computer Science, pp. 283-292, Lake Tahoe, Nev, USA, November 2006.

[30] H. Kong, E.-K. Teoh, J.-G. Wang, and R. Venkateswarlu, "Coplanar light sweep-surface supported uncalibrated photometric stereo," in Proceedings of the 17th International
Conference on Pattern Recognition (ICPR '04), vol. 4, pp. 9295, Cambridge, Mass, USA, August 2004.

[31] B. K. P. Horn, Robot Vision, MIT Press, Cambridge, Mass, USA, 1986.

[32] S. Chaudhuri and A. N. Rajagopalan, Depth from Defocus: A Real Aperture Imaging Approach, Springer, New York, NY, USA, 1999.

[33] V. Kolmogorov and R. Zabih, "What energy functions can be minimized via graph cuts?" IEEE Transactions on Pattern Analysis and Machine Intelligence, vol. 26, no. 2, pp. 147-159, 2004.

[34] V. Kolmogorov and R. Zabih, "Multi-camera scene reconstruction via graph cuts," in Proceedings of the 7th European Conference on Computer Vision-Part III (ECCV '02), vol. 2352 of Lecture Notes in Computer Science, pp. 82-96, Copenhagen, Denmark, May 2002.

[35] U. Mudenagudi, R. Singla, P. Kalra, and S. Banerjee, "Super resolution using graph-cut," in Proceedings of the 7th Asian Conference on Computer Vision-Part II (ACCV'06 ), vol. 3852 of Lecture Notes in Computer Science, pp. 385-394, Hyderabad, India, January 2006.

[36] Y. Boykov and V. Kolmogorov, "An experimental comparison of min-cut/max-flow algorithms for energy minimization in vision," IEEE Transactions on Pattern Analysis and Machine Intelligence, vol. 26, no. 9, pp. 1124-1137, 2004. 